\title{
Forensic, investigative and diagnostic microbiology: similar technologies but different priorities
}

\author{
Leah D Blondeau ${ }^{1}$, Joseph E Rubin ${ }^{1,2}$, Harry Deneer ${ }^{1,3}$, Rani Kanthan ${ }^{1,3}$, Stephen \\ Sanche ${ }^{1,4,5}$, Camille Hamula ${ }^{1,3,4}$ \& Joseph M Blondeau*,1,3,4,6,7 (iD) \\ ${ }^{1}$ University of Saskatchewan, Saskatoon, Saskatchewan, S7N 0W8, Canada \\ 2Department of Veterinary Medicine, University of Saskatchewan, Saskatoon, SK, S7N 0W8, Canada \\ ${ }^{3}$ Department of Pathology\& Laboratory Medicine, University of Saskatchewan, Saskatoon, SK, S7N OW8, Canada \\ ${ }^{4}$ Department of Clinical Microbiology, Royal University Hospital \& Saskatchewan Health Authority, University of Saskatchewan, \\ Saskatoon, SK, S7N 0W8, Canada \\ ${ }^{5}$ Department of Medicine, University of Saskatchewan, Saskatoon, SK, S7N 0W8, Canada \\ ${ }^{6}$ Department of Microbiology \& Immunology, University of Saskatchewan, Saskatoon, SK, S7N OW8, Canada \\ ${ }^{7}$ Department of Ophthalmology, University of Saskatchewan, Saskatoon, SK, S7NOW8, Canada \\ *Author for correspondence: Tel.: +1 306655 6943; Fax: +1 306655 6947; joseph.blondeau@saskhealthauthority.ca
}

"While forensic, investigative and diagnostic microbiology may focus on different priorities - the techniques, technology and approaches to working with a specimen are, for all intents and purposes, the same."

First draft submitted: 25 March 2019; Accepted for publication: 29 March 2019; Published online: 31 May 2019

Keywords: diagnostic $\bullet$ forensic $\bullet$ investigation $\bullet$ microbiology

The world of 'forensics' or 'forensic investigations' has been popularized by contemporary television programs such as Forensic Files and Crime Scene Investigation (CSI). The Canadian Oxford Dictionary defines forensic "as of or used in connection with courts of law, especially in relation to crime detection" and forensic science was defined as "the application of biochemical and or other scientific techniques to the investigation of crime" [1]. Indeed, the European Society for Clinical Microbiology and Infectious Diseases (ESCMID - www.escmid.org) established a study group on Forensic and Postmortem Microbiology with its vision and objectives to standardize microbiological sampling in forensic cases, create networks to facilitate cooperation among various experts working in forensic sciences, professional societies and between microbiologists from ESCMID and the European Network of Forensic Institutes.

While forensic, investigative and diagnostic microbiology may focus on different priorities - the techniques, technology and approaches to working with a specimen are, for all intents and purposes, the same. In forensics, the 'chain of custody' of the specimen is essential for ensuring a specimen (evidence) remains uncompromised and the findings can be used as evidence in criminal proceedings. In investigative and diagnostic microbiology, the importance of specimen identification and linkage to a specific patient is critical. For patients with moderate to severe infections, critical specimens (i.e., blood cultures, cerebral spinal fluid) giving rise to the correct identification of the infectious pathogen may profoundly impact morbidity and mortality. Misidentified specimens could lead to incorrect results for the wrong patient. In outbreaks, specimen collection and linkage to correct patients is critical in understanding the nuances of the outbreak and pinpointing the likely source.

Forensic microbiology is a newly emerging discipline that first gained global recognition as a result of the Bacillus anthracis attacks through the USA postal service in 2001 [2,3]. Indeed, with our own service during this time, suspicious packages/mails were handled with appropriate precautions in our diagnostic laboratory. It is an ideal tool in forensic investigations as microbial signatures are unique and can be isolated to specific environments. It can also be used to estimate postmortem interval (PMI) and link objects to owners [4]. While further research is 
required for this investigative technique, there are several instances of forensic microbiology in current research in which its importance in the forensic community is demonstrated, leading the way to future applications.

As summarized by Schmedes et al. [3], examples of microbial forensic and/or epidemiologic investigations with bacteria occurred in 2000-2001 with the characterization of Staphylococcus strains from drug paraphernalia [5], anthrax and related investigations [6,7], anthrax cases in Scotland [8], determination of the origin of the Haitian cholera outbreak in 2010 [9-11] and the trace origin for the Escherichia coli 0.104:H4 outbreak in Germany [12].

For investigations involving viral pathogens, HIV transmission from dentist to patient [13], intentional injections of HIV/HCV-contaminated blood into a partner [14], HCV infection of 275 patients by a Spanish anesthetist [15], the 2014 Ebola outbreak [16], examination of skeletal remains from World War II and parvovirus B19 characterization [17] are some notable examples. Arguably some of these investigations are less forensic-based as they were not all related to 'criminal' activity and, as such, may be more investigative microbiology with some instances possibly originating from diagnostic microbiology investigations.

Although microbial forensics can appear to be intimidating in nature, it requires much of the same processes that a forensic crime scene usually follows such as recognizing an event, containment to protect evidence integrity and maintaining a chain of custody, to name a few [18].

One main use in forensic microbiology is to determine a microbial signature for an agent [19]; an approach also used in outbreak investigations. This can allow tracing back to the original source, much like a fingerprint. Forensic microbiology also comes into effect when the body is decomposing [20]. It can be useful for determining the time of death from around 2-4 days and up to 25 days [20]. This also applies to deaths that occur in less than ideal conditions, such as extreme temperatures (cold or heat).

There is also evidence to suggest that forensic microbiology can be used to interpret cases of infectious diseases that result in sudden death, although this is also true of investigative and diagnostic microbiology. As deaths of this nature are a rarity in high income countries, it is often accompanied by a forensic autopsy to determine the exact cause of death [21]. An observational study by Morentin $e t$ al. determined the number of sudden deaths involving infectious diseases of patients from 1-34 years of age. Of 56 sudden unexpected deaths resulting from infection, examined from 1991 to 2010, the majority of patients died from myocarditis $(n=20)$, bronchopneumonia/pneumonia $(n=18)$, meningococcemia $(n=5)$ and viral pneumonia $(n=4)$ [21]. The authors concluded that while such deaths are infrequent (5\% of all deaths from infectious diseases), they do represent a significant portion of sudden deaths demonstrating the importance of forensic pathology investigation after death. One can question if it is truly forensic or investigational pathology or diagnostic microbiology considering a crime may not have been committed yet; however, the investigational technologies are the same.

Christoffersen reported on microbiological testing for establishing the cause of deaths in 42 forensic autopsies [22]. Interestingly, detailed descriptions of the microbiological technologies used were not described in any detail except to mention agar cultures for bacteria and fungi and PCR for viruses. A wide range of bacterial and viral pathogens were recovered and in some cases, in other words, 18/42 (42.8\%), the cause of death was classified as infectious (viral pneumonia, bacterial pneumonia, sepsis, meningitis, peritonitis and perforated appendicitis). For today's wellequipped clinical microbiology laboratory, isolation and identification of bacteria, yeast/fungus, viruses and parasites are routine from both living patients and postmortem patient specimens. VITEK ${ }^{\circledR} 2$ (or similar technologies, i.e, Microscan, AZ, USA), MALDI-TOF, Multi-plex PCR and $16 \mathrm{~S}$ ribosomal sequencing are routine technologies in our hospital and next-generation sequencing (NGS) and whole-genome sequencing (WGS) are being explored with NGS and WGS still being relatively more expensive and with increased turnaround time. Tagini and Greub reviewed WGS in clinical laboratories and indicated that challenges related to standardization, validation and procedural and bioinformatic pipelines were necessary to reach clinical standards [23]. To date, most applications have focused on outbreak investigations; however, virulence investigations and antimicrobial susceptibility testing have been explored. There is little doubt that the evolution of sequencing technology will significantly impact diagnostic microbiology laboratories.

In considering MALDI-TOF, spectrogram comparisons between bacterial isolates may be adequate for determining strain relatedness. Strejcek et al. compared whole-cell MALDI-TOF versus 16S, rRNA gene analysis for identification and dereplication of recurrent bacterial isolates [24]. They concluded that such an approach was efficient for dereplication of isolates for downstream analysis and with minimal loss of unique organisms. Additionally, they commented that MALDI-TOF analysis has potential for further improvement whereas 16S rRNA methodological limits have plateaued. Emami et al. reported that MALDI-TOF and 16S rRNA gene sequencing produced similar results at the genus level when comparing bacteria in ballast water [25]. Sato et al. evaluated 
repetitive PCR and MALDI-TOF for rapid strain typing of Bacillus coagulans and demonstrated that combined methods was advantageous for typing at both the genotype and phenotype levels [26]. Cronobacter (formerly Enterobacter) species was published as an emerging opportunistic foodborne pathogen associated with meningitis and necrotizing enterocolitis in infants [27]. Powdered infant formula appears to be the main source of Cronobacter species contamination. Lu et al. compared 75 strains of Cronobacter species by API 20E biochemical profiling, MALDI-TOF and genotype profiling by ribotype [27]. MALDI-TOF and biochemical identification were more accurate than ribotype analysis; however, MALDI-TOF and ribotyping showed higher discriminatory ability. The authors concluded MALDI-TOF has the potential to trace dissemination of Cronobacter along with production chains.

It is suggested that humans contain more bacterial cells in their body than they do of human cells [3]. Bacterial transfers appear to be more practical to examine on objects compared with DNA as DNA leaves only a small amount of residue, if any. Although circumstances like the CSI effect [28] tend to influence the public into believing that DNA is readily available, testable and reliable but this is often not the case, as DNA transfer would be quite minimal on object transfer compared with microorganisms. Our environments are not sterile nor are humans or animals; As such, bacterial (perhaps even yeast and fungal) transfer occurs regularly.

Microbial forensics can also be used to help determine location-based information of a person, to a certain extent [3]. It is argued that because microbial communities are complex in individuals, it can be very useful in determining the recent environment of victims, their diet or even in linking them back to other individuals based on the bacterial strain type(s) [3]. Scheidegger and Zimmerli commented on bacterial transmission in IV drug users [29] and Quagliarello et al. reported the similarities and differences in Staphylococcus aureus strains from drug-use networks by utilizing pulsed-field gel electrophoresis comparison of strains [5]. Strain characterization and comparisons can be utilized in sexual disease transmission cases, human trafficking cases or cases where an individual's last known location is undetermined.

As bioterrorism threats are a forefront area in microbial forensics, there is a stressed importance on real time diagnosis of potential threats. Bokhari discussed the positive outcomes of biosurveillance as a method of identifying and preventing future threats of bioterrorism attacks, or even accidental outbreaks [30]. The author goes on to state that this is a necessity as humans are more globally mobile now and a potential threat could be prevented through early identification methods. The author suggests the use of nanotechnology to develop biosensors designed for early identification of foreign pathogens; however, such applications will come with challenges.

Fierer $e t$ al. examined the presence of skin bacterial communities compared with objects touched by a person to see if the different bacterial communities can be specifically linked back [31]. This study took samples from three computer keyboards and compared samples taken from the fingerprints of their owners. The authors found that the bacterial communities on the objects can be distinguished between users and subsequently linked back to their owners with a high degree of certainty. Although further research is required, this could prove a useful forensic microbiology technique in cases absent of blood or tissue evidence and could further be an investigative microbiology approach to study organism or transmission outbreaks in noncriminal situations.

There is also evidence for microbes leaving a trail that can be used in forensic identification. Kupferschmidt discussed the examination of microbial samples and the capacity in which they can potentially be traced back to an individual, as microbial species are highly specific [32]. This article questions whether these microbial communities are significant enough to act as proof beyond reasonable doubt in forensic investigations, similarly to DNA evidence. Another doubt with this type of identification is that microbial communities can change over time [32]; a countermeasure to this is the potential development of a microbial bank similar to that of fingerprint identification records. The author acknowledges how difficult and impractical this is at this time but argues that, like the fingerprint database, it will take time to build up a library [32].

As previously stated, microbial forensics can be used to estimate PMI. As establishing PMI can be difficult, having another investigation tool alongside resources such as eyewitness testimonies can provide a more accurate time of death [33]. As information is not always available at the time of death, extra resources need to be utilized to ensure a more accurate PMI. Alternatively, microbes are always present at time of death no matter what environment the victim is located [33]; not only are they present, but they adapt to the environment located in predictable patterns as demonstrated in previous studies [33]. Further research on the environmental impact and all its variables is required.

Forensic microbiology is not only limited to circumstances involving crime scenes and deaths, but is also a tool useful in forensic counterfeit circumstances. One example includes a circumstance with counterfeit 'Colgate' brand toothpaste products in the USA and other countries in 2007 [34]. Some 200,000 tubes were removed for the market. 
The suspected products were gathered and experimental samples were isolated. Using 16S rDNA sequencing on the isolated samples, multiple bacteria were in the sample tubes including Pseudomonas species, Serratia species and Klebsiella species [34] and upward of $10^{6} \mathrm{CFU} / \mathrm{g}$ were recovered. These results were determined as posing a potential public health issue.

In the event of a bioterrorism attack, there needs to be a consideration for surge capacity [35]. Shapiro discussed the implications of being ill-prepared for surge capacity in microbiology labs in the event of a bioterrorism attack [36] and as such an argument can be made for more resources in preparation for such event. Suggestions for this include: offering incentives to encourage students to enroll in microbiology programs, increasing production of supplies needed for testing purposes and increasing the number of safety cabinets in labs. One large question relates to which laboratories could be prepared to handle such investigations. For example, a university-based medical center might be expected to have leading edge technology for diagnostic and research purposes and such technology gets used for patient care. Is it realistic to expect such laboratories to handle the investigation of bioterrorism threats or should that role be with federal/national or state/provincial public health laboratories? Additionally, trained clinical/medical microbiologists may lack formal or practical training related to the legal aspects of criminal investigation yet would be well versed in investigative and diagnostic microbiology.

It is important to reiterate that reliance on a single piece of evidence is impractical when looking for a culprit, as with any forensic investigation; microbial forensics is no different. Microbial evidence gathered should be used in conjunction with other pieces to prove beyond reasonable doubt if an instance occurred.

Investigative microbiology has practical applications. Concerns related to microbial contamination of contact lenses relates to the introduction of microorganisms to the eye and the potential for severe ocular disease including endophthalmitis. Land et al. reported on microbial contamination of counterfeit and unapproved decorative contact lenses [37]. Of the $300+$ lenses examined, $60 \%$ of counterfeit lenses and $27 \%$ of unapproved lenses had microbial contamination. Of 29 different brand products examined, nearly half (48\%) had at least one sample positive for microorganisms. Organism identification was by DNA sequencing. From counterfeit lenses, the following organisms were recovered: Pseudomonas species (including P. aeruginosa), Bacillus species, Stenotrophomonas species, Micrococcus species, Elizabethkingia species and others. Decorative lenses were found to have Paenibacillus species, Staphylococcus species, Bacillus species, Micrococcus species and others. In contrast, contamination rates on authentic lenses from the manufacturers had 2 of 54 (3.7\%) positive for microbial contamination with Bacillus and Micrococcus species. Associating organisms recovered from patients using contaminated lenses or from patients with infection would further close the loop showing potential clinical consequences.

Investigative microbiology is a regular occurrence in diagnostic clinical microbiology laboratories with technologies identical to those used in forensic investigations. As such, the terminologies of forensic or investigative microbiology appear interchangeable with the only real difference related to if the investigation is for criminal purposes or not. For diagnosing infections, proper specimen collection, transport to the laboratory, specimen labeling with unique patient identifiers and timely processing are of paramount importance. Similarly, with foodborne pathogen related outbreaks [38] - are these criminal (forensic) or investigative? In hospital-related outbreaks simply isolating an organism does not prove the outbreak, but a comparison of multiple strains from multiple patients for organism relatedness is a necessary standard [39].

Diagnostic laboratories inadvertently come into the criminal (forensic) arena when specimens related to potential cases of sexual abuse or sexual assault are processed. Indeed, the finding of a sexually transmitted organism, at a minimum, has important public health implications and perhaps is indicative of criminal activity. Dyson and Hosein published that detection of a sexually transmitted pathogen from a child is suggestive of sexual abuse and as such the diagnosis must be correct for clinical, social and legal reasons [40]. At the time of publication, the 'gold standard' of diagnosis was in vitro culture of the organism as the method offers maximum specificity; however, culture must be followed by appropriate confirmation testing. Hammerschlag and Guillen commented on the medical and legal implications in testing for sexually transmitted infections in children [41]. They also acknowledge that nucleic acid amplification tests (NAAT) have replaced culture methods for Neisseria gonorrhoeae in many laboratories. White NAAT assays offer improved sensitivity and noninvasive specimen collection. Such assays, like all assays, have limitations with $N$. gonorrhoeae [42-44] relating primarily to the observation that the gonococcus strains undergo genetic variation and recombination. With amplification assays, genetic variation may alter sequence target sites necessary for application and give rise to false negative results. $N$. gonorrhoeae may exchange genetic material with commensal Neisseria species [42-44]. For identification of $N$. gonorrhoeae after laboratory isolation, Buchanan et al. suggested MALDI-TOF be potentially used as a single method for identification in routine cases [45]. For 1090 
isolates, $91 \%$ were identified by MALDI-TOF after one analysis rising to $99.9 \%$ after two analyses, with a positive predictive value of $99.3 \%$. More data are likely necessary for strain comparison; however, Carannante $e t$ al. indicated that MALDI-TOF MS was a promising tool for real-time detection and cluster analysis for surveillance purposes of gonococcal strains [46]. Its use for criminal investigation remains to be determined but Spencer et al. have reported MALDI-TOF use for profiling trace constituents of condom lubricants in the presence of biological fluids [47].

Technologically advanced clinical microbiology laboratories have capabilities today that have never been previously recognized. For example, MALDI-TOF technology, $16 \mathrm{~S}$ ribosomal sequencing, limitless PCR-based assays, pulsed-field gel electrophoresis and now, NGS and WGS. There is little doubt on the expanding role of NGS and WGS and such technology will likely be a routine tool in diagnostic laboratories as its role in diagnostic and outbreak investigation continues to be defined. The isolation, detection and identification of microbes (pathogens or colonies) as well as the use of nucleic acid sequencing in advanced diagnostic laboratories means less of a need to refer specimens or organisms to external laboratories. Further advancements in technology will continue to equip many diagnostic laboratories with incredible capabilities for diagnostic, investigative and perhaps in some cases, forensic microbial investigations.

\section{Financial \& competing interests disclosure}

The authors have no relevant affiliations or financial involvement with any organization or entity with a financial interest in or financial conflict with the subject matter or materials discussed in the manuscript. This includes employment, consultancies, honoraria, stock ownership or options, expert testimony, grants or patents received or pending, or royalties.

No writing assistance was utilized in the production of this manuscript.

\section{References}

1. The Canadian Oxford Dictionary. Barber K (Ed.). Oxford University Press, ON, Canada (1998).

2. Lehman DC. Forensic microbiology. Clin. Lab. Sci. 25(2), 114-119 (2012).

3. Schmedes SE, Sajantila A, Budowle B. Expansion of microbial forensics. J. Clin. Microbiol. 54(8), 1964-1974 (2016).

4. Beans C. News Feature: Can microbes keep time for forensic investigators? Proc. Natl Acad. Sci. USA 115(1), 3-6 (2018).

5. Quagliarello B, Cespedes C, Miller M et al. Strains of Staphylococcus aureus obtained from drug-use networks are closely linked. Clin. Infect. Dis. 35(6), 671-677 (2002).

6. Keim PS, Budowle B, Ravel J. Microbial forensic investigation of the anthrax-letter attacks. In: Microbial Forensics. Breeze RG, Budowle B, Schutzer SE (Eds.). Elsevier Inc. New York, NY, USA15-25 (2011).

7. National Research Council. Review of the scientific approaches used during the FBI's investigation of the 2001 anthrax letters. The National Academic Press, Washingtonn, DC, USA. (2011).

8. Price EP, Seymour ML, Sarovich DS et al. Molecular epidemiologic investigation of an anthrax outbreak among heroin users, Europe. Emerg. Infect. Dis. 18(8), 1307-1313 (2012).

9. Chin CS, Sorenson J, Harris JB et al. The origin of the Haitian cholera outbreak strain. N. Engl. J. Med. 364(1), 33-42 (2011).

10. Hasan NA, Choi SY, Eppinger M et al. Genomic diversity of 2010 Haitian cholera outbreak strains. Proc. Natl Acad. Sci. USA 109(29), E2010-E2017 (2012).

11. Hendriksen RS, Price LB, Schupp JM et al. Population genetics of Vibrio cholerae from Nepal in 2010: evidence on the origin of the Haitian outbreak. mBio 2(4), e00157-e00111 (2011).

12. Grad YH, Lipsitch M, Feldgarden M et al. Genomic epidemiology of the Escherichia coli O104:H4 outbreaks in Europe, 2011. Proc. Natl Acad. Sci. USA 109(8), 3065 (2012).

13. Ou CY, Ciesielski CA, Myers G et al. Molecular epidemiology of HIV transmission in a dental practice. Science 256(5060), 1165-1171 (1992).

14. Metzker ML, Mindell DP, Liu XM, Ptak RG, Gibbs RA, Hillis DM. Molecular evidence of HIV-1 transmission in a criminal case. Proc. Natl Acad. Sci. USA 99(22), 14292-14297 (2002).

15. Gonzalez-Candelas F, Bracho MA, Wrobel B, Moya A. Molecular evolution in court: analysis of a large hepatitis C virus outbreak from an evolving source. BMC Biol. 11, 76 (2013).

16. Gire SK, Goba A, Andersen KG et al. Genomic surveillance elucidates Ebola virus origin and transmission during the 2014 outbreak. Science 345(6202), 1369-1372 (2014).

17. Toppinen M, Perdomo MF, Palo JU et al. Bones hold the key to DNA virus history and epidemiology. Sci. Rep. 5, 17226 (2015).

18. Microbial Forensic. Breeze R, Budowle B, Schutzer SE (Eds). Elsevier/Academic Press, Boston, MA, USA (2005).

19. Lehman DC. Forensic microbiology. Clin. Lab. Sci. 25(2), 114-119 (2012). 
20. Metcalf JL, Carter DO, Knight R. Microbiology of death. Curr. Biol. 26(13), R561-R563 (2016).

21. Morentin B, Suárez-Mier MP, Aguilera B, Arrieta J, Audicana C, Fernández-Rodríguez A. Clinicopathological features of sudden unexpected infectious death: population-based study in children and young adults. Forensic Sci. Int. 220(1), 80-84 (2012).

22. Christoffersen S. The importance of microbiological testing for establishing cause of death in 42 forensic autopsies. Forensic Sci. Int. 250, 27-32 (2015).

23. Tagini F, Greub G. Bacterial genome sequencing in clinical microbiology: a pathogen-oriented review. Eur. J. Clin. Microbiol. Infect. Dis. 36(11), 2007-2020 (2017).

24. Strejcek M, Smrhova T, Junkova P, Uhlik O. Whole-cell MALDI-TOF MS versus $16 S$ rRNA gene analysis for identification and dereplication of recurrent bacterial isolates. Front. Microbiol. 9, 1294 (2018).

25. Emami $\mathrm{K}$, Askari $\mathrm{V}$, Ullrich $\mathrm{M}$ et al. Characterization of bacteria in ballast water using MALDI-TOF mass spectrometry. PLoS ONE 7(6), e38515 (2012).

26. Sato J, Nakayama M, Tomita A, Sonoda T, Hasumi M, Miyamoto T. Evaluation of repetitive-PCR and matrix-assisted laser desorption ionization-time of flight mass spectrometry (MALDI-TOF MS) for rapid strain typing of Bacillus coagulans. PLoS ONE 12(10), e0186327 (2017).

27. Lu Y, Chen Y, Lu XA et al. Comparison of methods for the microbiological identification and typing of Cronobacter species in infant formula. J. Dairy Sci. 97(2), 632-641 (2014).

28. Ley BL, Jankowski N, Brewer PR. Investigating CSI: portrayals of DNA testing on a forensic crime show and their potential effects. Public Underst. Sci. 21(1), 51-67 (2012).

29. Scheidegger C, Zimmerli W. Infectious complications in drug addicts: seven-year review of 269 hospitalized narcotics abusers in Switzerland. Rev. Infect. Dis. 11(3), 486-493 (1989).

30. Bokhari H. Exploitation of microbial forensics and nanotechnology for the monitoring of emerging pathogens. Crit. Rev. Microbiol. 44(4) 504- 521 (2018).

31. Fierer N, Lauber CL, Zhou N, McDonald D, Costello EK, Knight R. Forensic identification using skin bacterial communities. Proc. Natl Acad. Sci. USA 107(14), 6477-6481 (2010).

32. Kupferschmidt K. A trail of microbes. Science 351(6278), 1136-1137 (2016).

33. Metcalf JL, Xu ZZ, Bouslimani A, Dorrestein P, Carter DO, Knight R. Microbiome tools for forensic science. Trends Biotechnol. 35(9), 814-823 (2017).

34. Brzezinski JL, Craft DL. Characterization of microorganisms isolated from counterfeit toothpaste. J. Forensic Sci. 57(5), 1365-1367 (2012).

35. Rubinson L, Nuzzo JB, Talmor DS, O’Toole T, Kramer BR, Inglesby TV. Augmentation of hospital critical care capacity after bioterrorist attacks or epidemics: recommendations of the Working Group on Emergency Mass Critical Care. Crit. Care Med. 33(10), 2393-2403 (2005).

36. Shapiro DS. Surge capacity for response to bioterrorism in hospital clinical microbiology laboratories. J. Clin. Microbiol. 41(12), 5372-5376 (2003)

37. Land AD, Penno KL, Brzezinski JL. Identification of microorganisms isolated fromcounterfeit and unapproved decorative contact lenses. J. Forensic Sci. 63(2), 635-649 (2018).

38. Cavanagh K, Johnstone T, Huhtinen E et al. Foodborne illness outbreak investigation in a high-profile sports club. Sports Med. Open 3(1), 24 (2017).

39. Nutman A, Marchaim D. 'How to do it' - molecular investigation of a hospital outbreak. Clin. Microbiol. Infect. 25(6), 688-695 (2018).

40. Dyson C, Hosein IK. The role of the microbiology laboratory in the investigation of child sexual abuse. J. Med. Microbiol. 45(5), 313-318 (1996).

41. Hammerschlag MR, Guillen CD. Medical and legal implications of testing for sexually transmitted infections in children. Clin. Microbiol. Rev. 23(3), 493-506 (2010).

42. Tabrizi SN, Chen S, Cohenford MA et al. Evaluation of real-time polymerase chain reaction assays for confirmation of Neisseria gonorrhoeae in clinical samples tested positive in the Roche Cobas Amplicor assay. Sex. Transm. Infect. 80(1), 68-71 (2004).

43. Whiley DM, Tapsall JW, Sloots TP. Nucleic acid amplification testing for Neisseria gonorrhoeae: an ongoing challenge. J. Mol. Diagn. 8(1), 3-15 (2006).

44. Whiley DM, Garland SM, Harnett G et al. Exploring 'best practice' for nucleic acid detection of Neisseria gonorrhoeae. Sexual Health 5(1), 17-23 (2008).

45. Buchanan R, Ball D, Dolphin H, Dave J. Matrix-assisted laser desorption-ionization time-of-flight mass spectrometry for the identification of Neisseria gonorrhoeae. Clin. Microbiol. Infect. 22(9), 815.e815-815.e817 (2016).

46. Carannante A, De Carolis E, Vacca P et al. Evaluation of matrix-assisted laser desorption ionization-time of flight mass spectrometry (MALDI-TOF MS) for identification and clustering of Neisseria gonorrhoeae. BMC Microbiol. 15, 142 (2015).

47. Spencer SE, Kim SY, Kim SB, Schug KA. Matrix-assisted laser desorption/ionization-time of flight-mass spectrometry profiling of trace constituents of condom lubricants in the presence of biological fluids. Forensic Sci. Int. 207(1-3), 19-26 (2011). 
